\title{
Diagnostics and Prediction of Glaucoma in Patients with Familial Congenital Iris Hypoplasia
}

\author{
Tatiana Iureva',2, Andrey Shchuko', Yulia Pyatova1 \\ ${ }^{1}$ Irkutsk Branch, S. Fyodorov Eye Microsurgery Federal State Institution, Irkutsk, Russia \\ ${ }^{2}$ Irkutsk State Medical Academy for Postgraduate Education, Irkutsk, Russia \\ Email: tnyurieva@mail.ru
}

Received 7 July 2015; accepted 11 August 2015; published 14 August 2015

Copyright (C 2015 by authors and Scientific Research Publishing Inc.

This work is licensed under the Creative Commons Attribution International License (CC BY). http://creativecommons.org/licenses/by/4.0/

c) (7) Open Access

\section{Abstract}

Purpose: To identify the clinical features of the syndrome Frank-Kamenetsky and determine the criteria of early formation of glaucoma. Materials and Methods: We observed 52 patients. Follow up period was from 5 to 22 years. The first group (juvenile) consisted of males who had the first signs of glaucoma diagnosed before the age of $12(n=22)$. The average age of the group was $10.1 \pm$ 2.4 years. The control group included healthy males $(n=30)$ in the same age range (average age $7.2 \pm 1.6$ years). The second group (adults) consisted of patients who had the first signs of glaucoma diagnosed after the age of 18 and elder. The average age of the group was $32.44 \pm 6.28$ years. The control group had males $(n=30)$ in the same age range (average age $26.59 \pm 4.12$ years). The inclusion criterion was: the presence of congenital bilateral mesodermal iris leaf hypoplasia, trabecular dysgenesis signs, the presence of blood relatives on the maternal line (grandfather, uncle) male with similar changes iridociliary zone and glaucoma. Criteria of glaucoma formation were: increased IOP more than $21 \mathrm{mmHg}$ with accompanying it expansion of the cup/disc ratio, reducing the thickness of the nerve fiber layer (RNFL) according to OCT. Results: It was found that FrankKamenetsky Syndrome had an X-linked with sex, recessive inheritance and was characterized by bilateral congenital irisdysgenesis and goniodysgenesis with the accession glaucoma. Predictors of glaucoma formation in early childhood are a combination of: 1) congenital subtotal atrophy of iris mesodermal layer (from 0 to $30 \mathrm{mkm}$ ) with signs of progressive dystrophy; 2) nonprogressive congenital megalocornea (cornea diameter 12 - 14 mm); 3) iridotrabecular dysgenesis of II-III degree; 4) hyperopic refraction in axial myopia.

\section{Keywords}

Glaucoma, Megalocornea, Congenital Mesodermal Iris Atrophy, Goniodysgenesis 


\section{Introduction}

To our knowledge, this syndrome was first described in Russia in 1925, was characterized by congenital atrophy of the mesodermal layer of the iris and goniodysgenesis and led to the development of glaucoma [1]-[3]. It was later named as Frank-Kamenetsky Syndrome in honor of the discoverer. This syndrome is different from "congenital hypoplasia of the iris stroma" because it is an X-linked recessive as opposed to autosomal dominant in its inheritance. In addition, the long-term (over 20 years) patient follow up period allowed revealing of features for the syndrome and glaucoma [4]-[6].

In a retrospective study of medical records, it is found that the formation of glaucoma in these patients occurs either in a child aged from 0 to 12 years or in an adult in their second or third decade of life. The cause of congenital glaucoma formation changes in iridocorneal angle, "cog” fixing of the iris and the gray veil mesodermal tissue inclusions in the trabecular zone. In contrast to other forms of congenital glaucoma, forming of buphthalmos and acute IOP decompensation never occurs. Glaucoma occurs as open angle form, which makes it difficult to diagnose in the early stages of development. It is suggested that the mechanisms of glaucoma development in different ages can have fundamental differences. These differences are dependent on the combination of several characteristics of mesenchymal dysgenesis inherited in this syndrome.

\section{Purpose}

To identify the clinical features of the Frank-Kamenetsky syndrome and determine the criteria of early formation of glaucoma

\section{Materials and Methods}

The study was conducted in accordance with the Declaration of Helsinki and approved by the Ethics Committee of the institution. All patients signed an Informed Consent form prior to participation in the study.

\section{Subjects}

52 patients (males) with Frank-Kamenetsky syndrome. The control group included 60 patients (males).

Therefore, in the future, all patients were divided into two groups according to their glaucoma development time to identify specific symptoms and patterns of disease formation:

1) The first group (juvenile), consisted of males who had the first signs of glaucoma diagnosed before the age of $12(n=22)$. The average age of the group was $10.1 \pm 2.4$ years. The control group included healthy males $(\mathrm{n}=30)$ in the same age range (average age $7.2 \pm 1.6$ years).

2) The second group (adults), consisted of patients who had the first signs of glaucoma diagnosed after the age of 18 and elder. The average age of the group was $32.44 \pm 6.28$ years. The control group were males $(n=30)$ in the same age range (average age $26.59 \pm 4.12$ years).

The inclusion criterion were: the presence of congenital bilateral mesodermal iris leaf hypoplasia, trabecular dysgenesis signs, the presence of blood relatives on the maternal line (grandfather, uncle) male with similar changes iridociliary zone and glaucoma. Criteria of glaucoma formation were: increased IOP more than 21 mmHg with accompanying it expansion of the cupldisc ratio, reducing the thickness of the nerve fiber layer (RNFL) according to OCT.

\section{Ophthalmological Examination}

Ophthalmological examination included standard methods of diagnosis (visual acuity measurement, biometry, refractometry, ophthalmoscopy, gonioscopy) as well as examinations of morphology iris and optic nerve-optical coherence tomography (Cirrus HD-OCT, Carl Zeiss Meditec Inc., USA), ultrasound biomicroscopy imaging (UBM-840, Hamphrey).

\section{Statistical Analysis}

The statistical data on all the studied parameters was represented as the mean value \pm standard deviation. The difference between the treatment groups and control group was defined using Mann-Whitney U-test. The critical level of significance P upon the examination of statistical hypotheses was 0.05 . All the calculations were made 
using the program STATISTICA 8.0, Stat Soft. Inc., USA.

\section{Results}

\subsection{The Study of the Iris.}

In the process of examination and long-term follow up of the patients with the syndrome, it was found that the symptomatic feature is a peculiar congenital hypoplasia of the iris stroma with exposure of its pigment epithelium. In addition this is always a bilateral process (i.e. both eyes are affected). While the defect of the front mesodermal iris layer is congenital, the destruction of the backsheet is acquired, appearing later in life and progressing throughout. External changes in the iris are so consistent and typical that after the examination of the patients it seems that they are close relatives or brothers (Figure 1).

While the normal pupillary zone [7] of an iris is darker than a ciliary zone, all patients with the syndrome have thicker, light gray or yellow, lackluster pupillary zone, devoid of the normal luster. The periphery of the iris looks like a wide ring of contrasting brown or blue-purple. The reason for the color contrast is the hypoplasia of the iris stroma, through which the pigment epithelium is seen.

In $44.4 \%$ of the cases of the patients in the juvenile group, and in $6.8 \%$ of the cases of the patients in the adult group in addition to the two-color staining there was also rough damage of the iris:

1) Iridoschizis and radial zones of the transillumination around the iris periphery.

2) Slotted through-defects of the iris in the ciliary zone (Figure 2).

3) Polycoria (Figure 3).

4) Ectopia and pupillary ring deformation (Figure 4).

Long-term iris monitoring in patients of this group has revealed that the iris abnormalities are the successive stages of the progressive atrophy of the stroma and the destruction of the pigment epithelium.

The study of the iris by the OCT method [8] confirmed the presence of rough congenital bilateral hypoplasia of the stroma in patients of the juvenile group (Figure 5). In the control group, the thickness of the iris stroma was from 460 to 283 microns. The iris stroma of the juvenile group was severely thinner, up to its complete absence in the ciliary zone (from $126.0 \mathrm{mkm}$ to $0 \mathrm{mkm}$ ) and was 3 - 5 times thinner than that of healthy children. In the adult group, the thickness of the iris stroma was also thinner and it was on average $209.27 \pm 44.27 \mathrm{mkm}$ $(P=0.001)$. The pigment layer in all patients with the syndrome was dramatically thickened, up to 70 microns at the limbus and up to 90 microns in the pupil area, which is almost 1.5 times higher than normal (Figure 6).

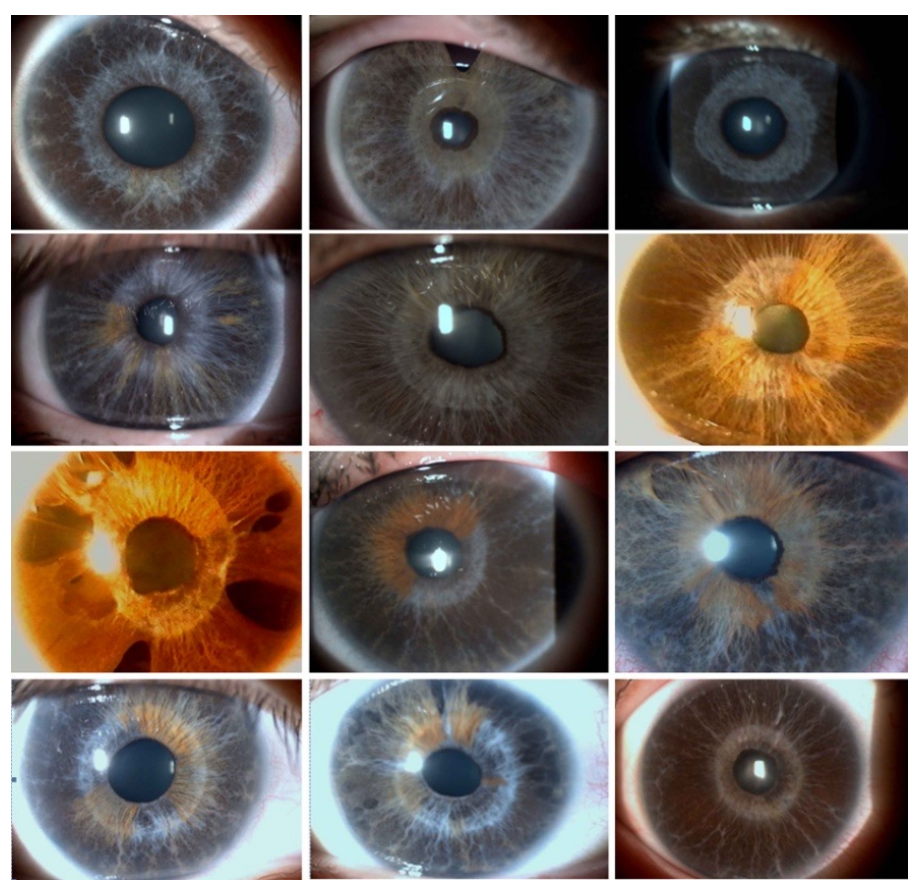

Figure 1. Photos of iris of 12 patients of the juvenile group. 


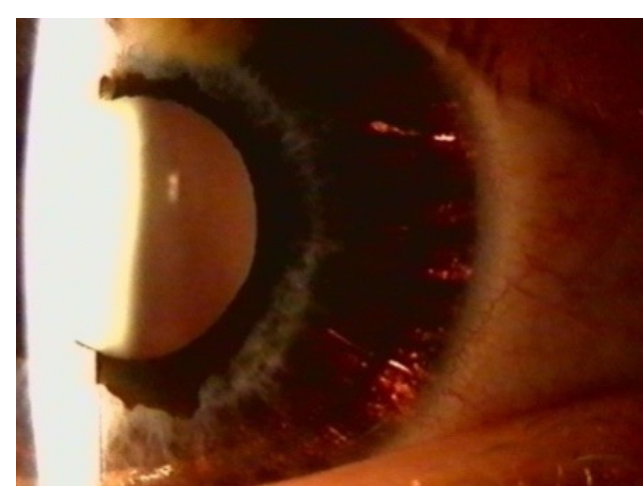

Figure 2. Radial zones of transillumination around the periphery of iris.

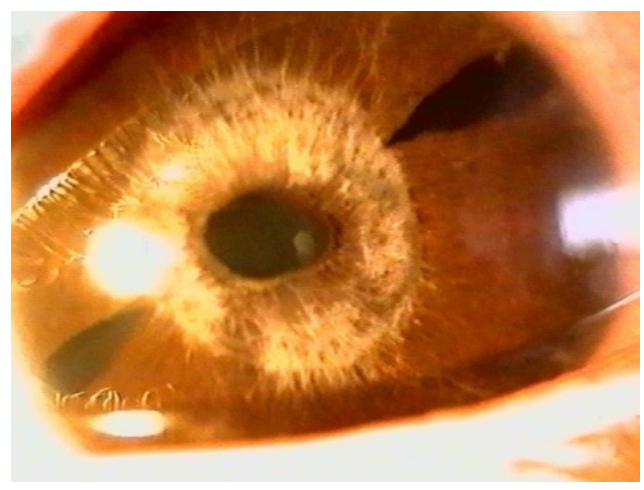

Figure 3. Large triangular breaks of the iris.

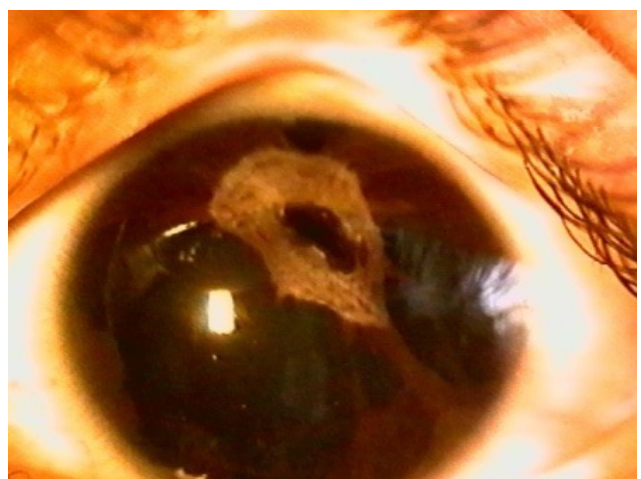

Figure 4. Ectopia of the pupil, large iris breaks.

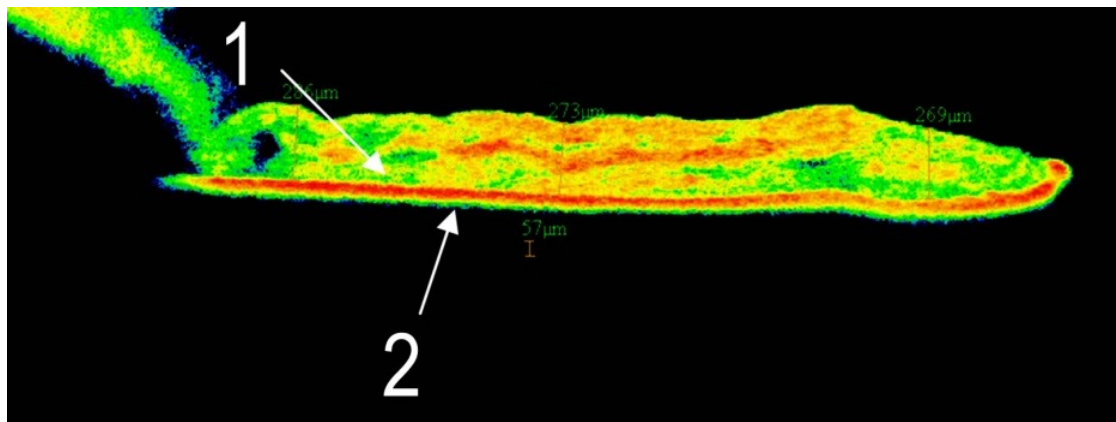

Figure 5. OCT imaging of the iris in healthy eyes: 1-stroma 236 - 405 microns, 2pigment epithelium 57 - 60 microns. 


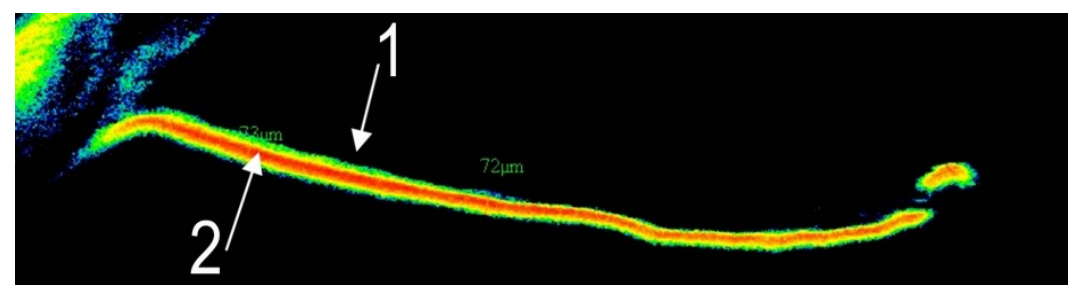

Figure 6. OCT imaging of the iris of a 6 year-old patient with glaucoma: 1 -iris stroma -0 micron, 2 - pigment epithelium—72 - 78 microns.

The smallest thickness of the pigment layer was registered next to the iris through-defects. That is, with the progression of the process there is rupture and wrinkling of the tissues, rather than atrophy of the tissue.

\subsection{Features of the Iridocorneal Angle}

Goniodysgenesis is characterized by the front fastening of the iris above the trabecular meshwork or fastening of the iris to the modified anterior border of the Schwalbe's ring, which was protruding into the anterior chamber in the form of the crest and was characterized as a posterior embryotoxon. Posterior embryotoxon was detected in patients of the juvenile group in 100\% of the cases (Figure 7).

The degree of goniodysgenesis (classification Hoskins HD Jr., Shaffer RN: 1-Trabeculodysgenesis, 2-partial iridotrabekulodysgenesis, 3-full iridotrabekulodysgenesis)), evaluated by a point system, in this group of patients juvenile group was $2.1 \pm 0.71$, in individuals of adult group $1.19 \pm 0.04$, which significantly exceeds the control group $-0.05 \pm 0.22$ and $0.07 \pm 0.26$ respectively $(P=0.004$ and $P=0.05)$. Despite the fact that such a condition of the anterior chamber angle should lead to a complete pre-trabecular meshwork retention, acute decompensation of IOP in patients was not observed, which is very different for this form of glaucoma in comparison with simple congenital glaucoma. Perhaps this can be explained by the hypoplasia of iris stroma in patients with the syndrome when the partial drainage of aqueous humor is preserved. It was found that the through-defects of an iris are formed at the initial stromal thickness of up to 30 microns.

\subsection{Cornea}

It was found that the clinically significant corneal pathology [9] [10] was diagnosed in the majority of the juvenile group patients. There was an increase in congenital corneal diameter of more than $12 \mathrm{~mm}$ (Figure 8) with normal values of intraocular pressure in 20 of the researched cases, which determined a significant difference ( $F$-criterion-4.33 with a significance level of $P=0.05$ ) on the basis of the control group. This condition was diagnosed as megalocornea.

Corneal thickness of patients with megalocornea averaged $591.57 \pm 52.67 \mathrm{mkm}$, resulting in an increase in the average thickness of the cornea in juvenile group patients of 50 microns, compared with healthy children.

In addition, it was found that the increase of the cornea diameter in patients born with the Frank-Kamenetsky Syndrome is detected at birth, is independent of the IOP level, is not progressive and, as opposed to simple congenital glaucoma, is accompanied by thickening (not thinning) of the cornea. Therefore, such cornea condition is one of the manifestations of congenital malformations of the eye's mesenchymal tissue.

Due to the fact that mesenchymal dysgenesis involves changing of all structures of the anterior segment of an eye, the following was studied in great detail: parameters of the cornea, lens and anterior chamber angle values, changes in the ways of the outflow of aqueous humor, the state of the iris at all stages of the disease (Table 1).

\subsection{Discriminant Analysis of the Study's Results of the "Juvenile" and "Adult" Groups of Patients}

Informative attributes, calculated from the F-Fisher criterion, are distributed as follows (Table 2). The most informative attributes were the thickness of the iris stroma (F-score 22.1) and the degree of goniodysgenesis (F-score 22.16), with a significance level of $P<0.00001$. It was observed that such features as the thickness of the cornea (the F-score 6.16) and the diameter of the cornea (F-criterion 1.9) appeared to have the same degree of canonical value construction with $P<0.05$. In addition, the discriminant analysis revealed the possible impact indicators such as the degree of refraction (F-score 3.2 for $P=0.07$ ), the length of an eyeball (F-score 5.64), the 


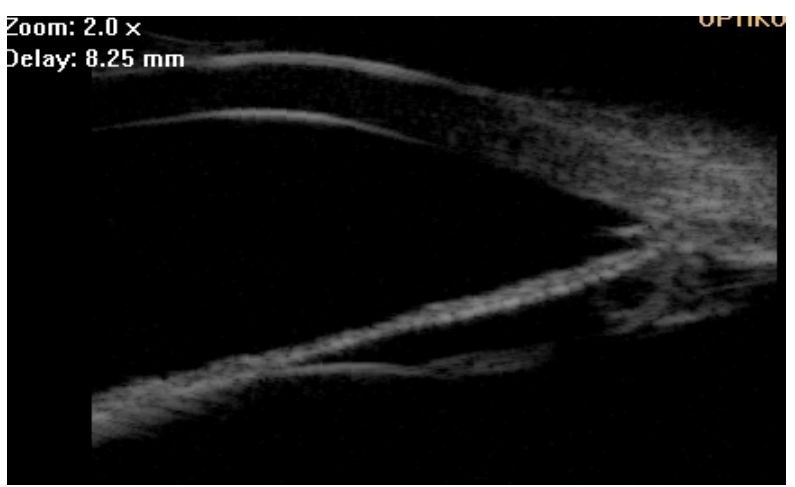

Figure 7. UBM of the anterior segment of the eye of Patient K. In the top of the anterior chamber angle-protrusion of an anterior border of the Schwalbe's ring-posterior embryotoxon.

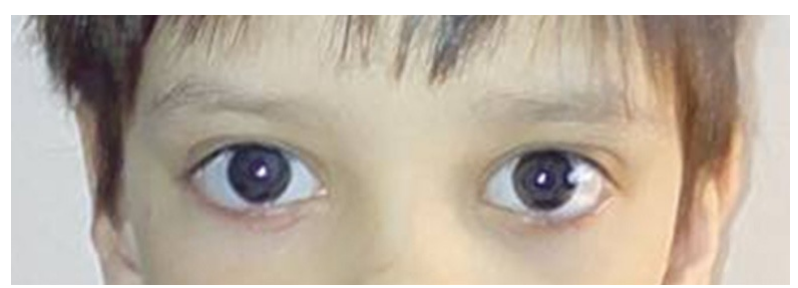

Figure 8. Photo of the patient with a corneal diameter of $14 \mathrm{~mm}$.

Table 1. Results of a comparative analysis of the parameters of the visual system of patients with the syndrome and the control group $(\mathrm{M} \pm \mathrm{s})$.

\begin{tabular}{|c|c|c|c|c|c|}
\hline Data & $\begin{array}{c}1 \text {-control group, } \mathrm{n}= \\
30 \text {, males, average age } \\
7.2 \pm 1.6\end{array}$ & $\begin{array}{l}\text { 2-“juvenile" group, } \mathrm{n}= \\
\text { 22, males, average age } \\
10.1 \pm 2.4\end{array}$ & $\begin{array}{c}\text { 3-control group, } n= \\
\text { 30, males, average age } \\
26.59 \pm 4.12\end{array}$ & $\begin{array}{l}\text { 4-“adult” group, } \mathrm{n}= \\
\text { e } 28 \text {, males, average age } \\
32.44 \pm 6.28\end{array}$ & $\begin{array}{c}P, \\
\text { Mann-Whitney }\end{array}$ \\
\hline Refraction of an eye & $+0.4 \pm 0.28$ & $+0.41 \pm 1.75$ & $-0.16 \pm 0.67$ & $-0.72 \pm 1.58$ & $3-4=0.008$ \\
\hline Diameter of the cornea, mm & $10.32 \pm 0.40$ & $12.48 \pm 0.96$ & $10.18 \pm 0.39$ & $10.83 \pm 0.67$ & $1-2=0.045$ \\
\hline CCT, microns & $546.28 \pm 37.94$ & $591.57 \pm 52.67$ & $558.94 \pm 33.22$ & $548.63 \pm 38.09$ & $1-2=0.007$ \\
\hline $\begin{array}{l}\text { Thickness of the iris stroma, } \\
\text { microns ( OCT) }\end{array}$ & $334.57 \pm 30.12$ & $100.57 \pm 27.25$ & $328.94 \pm 30.20$ & $209.27 \pm 44.27$ & $\begin{array}{l}1-2=0.001 \\
3-4=0.001\end{array}$ \\
\hline $\begin{array}{l}\text { Thickness of the iris pigment } \\
\text { epithelium, microns (OCT) }\end{array}$ & $62.75 \pm 5.54$ & $72.61 \pm 7.58$ & $62.11 \pm 5.54$ & $79.52 \pm 6.75$ & $\begin{array}{l}1-2=0.006 \\
3-4=0.004\end{array}$ \\
\hline $\begin{array}{l}\text { Availability through-defects } \\
\text { of the iris, \% }\end{array}$ & - & 44.4 & - & 6.8 & $\begin{array}{l}1-2=0.002 \\
3-4=0.002\end{array}$ \\
\hline Degree of a goniodisgenesis & $0.05 \pm 0.22$ & $2.10 \pm 0.71$ & $0.07 \pm 0.26$ & $1.19 \pm 0.40$ & $\begin{array}{l}1-2=0.004 \\
3-4=0.05\end{array}$ \\
\hline
\end{tabular}

magnitude of the anterior chamber (F-criterion 3, 3 for $P=0.07$ ).

Therefore, the most important signs determining the formation of glaucoma in children are congenital subtotal dysplasia of the iris stroma, goniodysgenesis and corneal changes by type of megalocornea characterized by increase of the diameter and CCT of the cornea. Other signs altogether characterize the discrepancy between the length of the eyeball and the degree of refraction, because myopic component in these patients is compensated by a weak refractive power of the cornea with its congenital dysgenesis.

\subsection{The Types of Inheritance of the Gene}

The conducted Genealogical Analysis showed that all of the patients were Caucasian and did not have any other somatic or eye diseases [11] [12]. During the cytogenetic examination of the probands in families A, B, and C (pedigrees \#1, 2, 3) no chromosomal abnormalities were found, which may indicate the monogenetic nature of the disease. The most common ways of inheritance of the Frank-Kamenetsky Syndrome are seen in the P. family (Chart 1). 


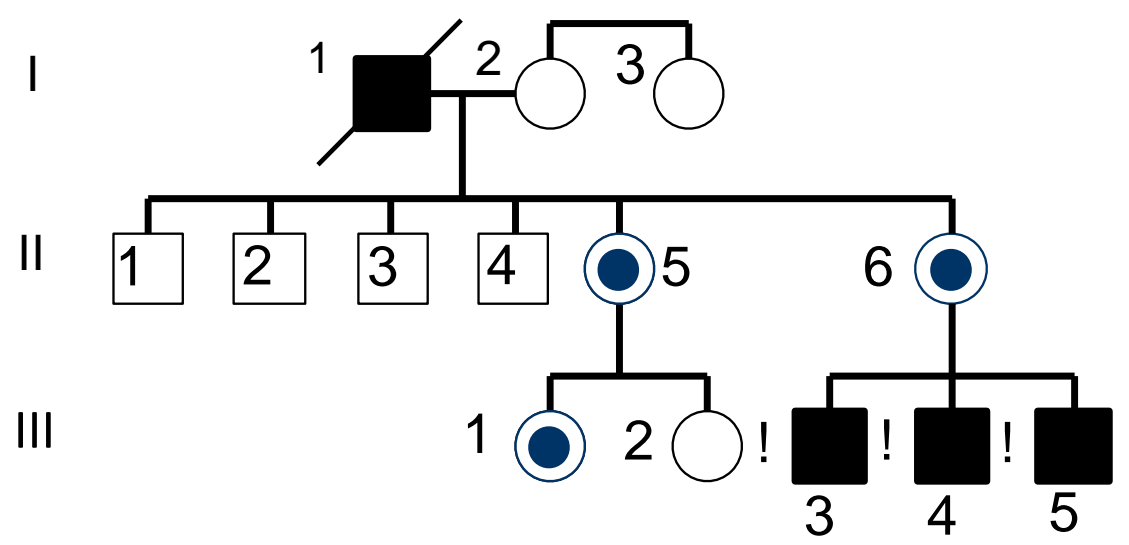

Chart 1.

I (1) — proband, died blind at 58 years old.

II (5)—no eye pathology, 37 years old.

II (6) — no eye pathology, 43 years old.

III (3)—proband-1, glaucoma since age 5.

III (4)—sibs, glaucoma since age 21.

III (5)—sibs, no glaucoma, 28 years old.

Female, carrier of pathological gene (carrier)_-

Healthy Female-

Individual with Frank-Kamenetsky Syndrome-

Healthy Male-

According to the provided genealogical Chart 1, 3 boys with congenital hypoplasia of the iris are observed within the P. family. The oldest brother's glaucoma was diagnosed at the age of 5, the middle brother's IOP began to increase at the age of 21, and the younger brother had a phenotypic syndrome only at the age of 28. Their maternal grandfather was blind from glaucoma, and died at the age of 58. Proband's and sibling's mother does not have any vision problems but has micro signs of the disease.

Thus, the mechanism of inheritance of pathological signs in this syndrome corresponds to x-linked recessive manner on the following criteria:

1) Common in men.

2) All female children of an affected father will be carriers. (Daughters possess their father's X-chromosome in $100 \%$ cases). The sons of the daughter will have a $50 \%$ probability to be affected.

3) No male children of the sick father will be affected. (Sons do not inherit their father's X-chromosome).

4) Heterozygous females are considered carriers and generally will not manifest clinical symptoms of the disease, but some of them may have varying degrees of severity of the disease.

The presence of such micro phenotypic symptoms of the syndrome can be taken into consideration in a prenatal genetic diagnosis.

\section{Discussion}

According to the study results, the initial mesodermal layer thickness of the iris at birth is a sign of dysgenesis, which is directly correlated with the degree of goniodysgenesis, abnormalities of the cornea, and the presence of partial or complete posterior embryotoxon. In general, alteration of the iris may determine the degree of anterior segment dysgenesis [13]-[15]. Formation of glaucoma in children is caused by a combination of congenital abnormalities of the cornea, iris, and the iridocorneal angle, as well as the presence of coarse dysgenesis of the anterior segment of the eye. These are, namely, the association of subtotal hypoplasia of the iris's mesodermal sheet (thickness from 0 to $30 \mathrm{mkm}$ ) with symptoms of progressive dystrophy, congenital megalocornea, posterior embryotoxon, and dysgenesis of iridocorneal angle of II-III degree.

Identification of the criteria and mechanisms of glaucoma is important as it allows not only to diagnose the disease at early preclinical stages of development but also to assign pathogenetically-based treatment soon 
Table 2. Classification features of the syndrome of the juvenile group.

\begin{tabular}{lcccc}
\hline & Data & \% contribution & F-criterion & $P$-level \\
\hline X7 & Thickness of the iris stroma & $25.25 \%$ & 66.61 & 0.00001 \\
X6 & Degree of goniodysgenesis & $19.85 \%$ & 22.16 & 0.00001 \\
X4 & Thickness of the cornea & $11.76 \%$ & 5.64 & 0.034 \\
X5 & Diameter of the cornea & $6.50 \%$ & 6.16 & 0.03 \\
X1 & Magnitude of the anterior chamber & $8.16 \%$ & 3.2 & 0.07 \\
X3 & Refraction of the eye & $8.66 \%$ & 3.3 & 0.071 \\
X2 & Length of an eyeball & $11.11 \%$ & 3.2 & 0.07 \\
\hline
\end{tabular}

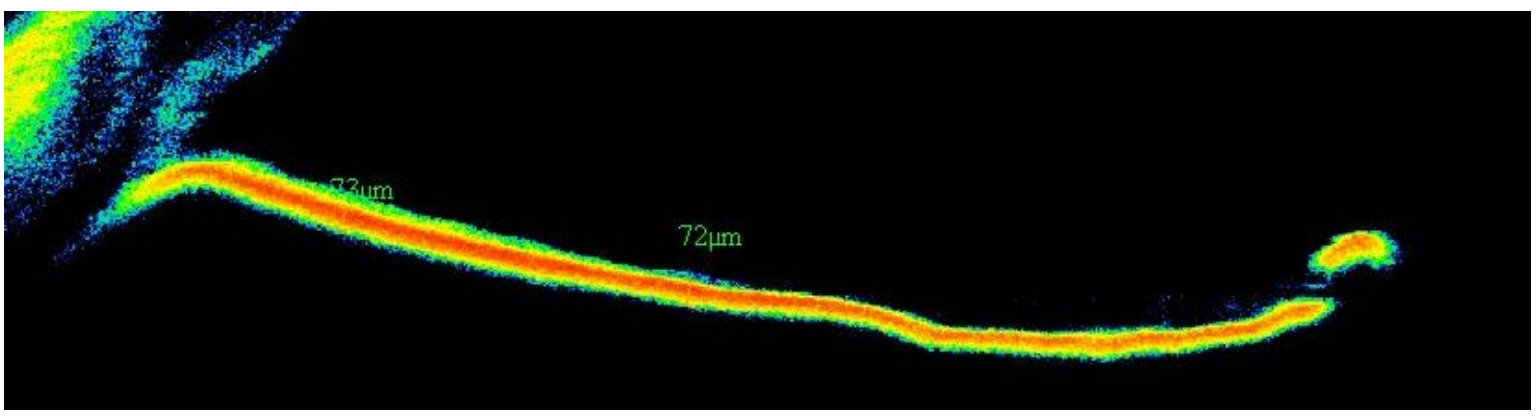

(a)

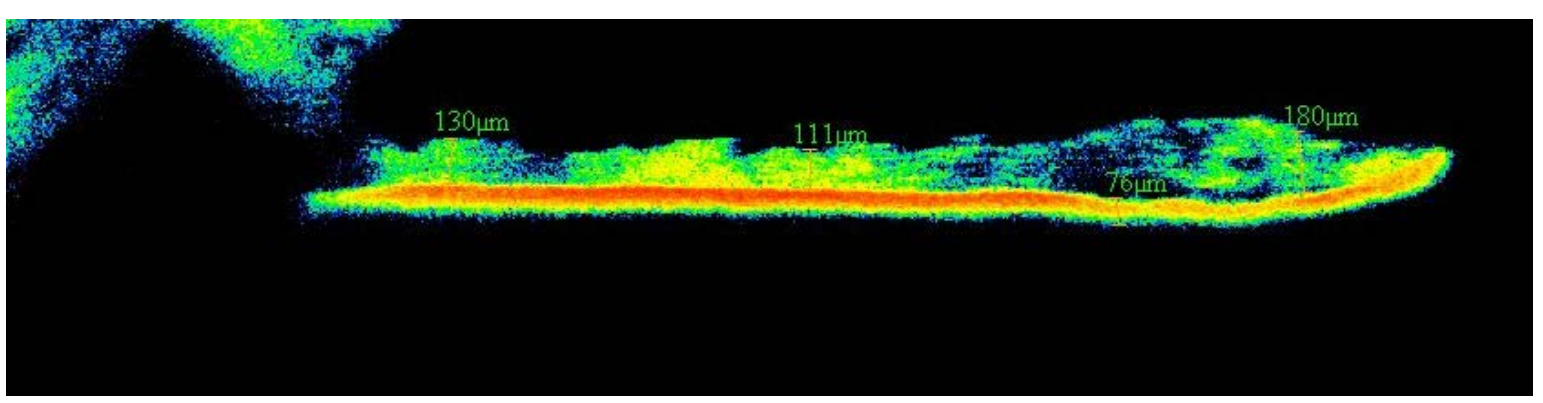

(b)

Figure 9. (a) OCT of the iris of Patient D., 11 years old. Stroma 0. Pigment layer (1) 72 - 78 mkm, (b) OCT of the iris of D.’s brother, 14 years old, Stroma 111 - 180 mkm (2). Pigment layer (1) 76 mkm, compacted, transparency reduced.

enough. The special importance in juvenile patients with glaucoma is the diminished visual function for several reasons. First, the development of glaucoma is an asymptomatic process, therefore an ophthalmologist is not involved until a later stage of the disease progression. Second, antihypertensive drug therapy has a weak hypotensive effect on this type of glaucoma. Third, is the progressive destruction of the iris, leading to impairment of accommodation and diaphragm function, which causes light scattering and further reduces a patient's visual acuity. Pathological process usually ends in late detection of glaucoma with blindness or poor vision at 40 - 50 years of age.

The combination of moderate iris hypoplasia with gonidysgenesis of I degree causes formation of glaucoma after the age of 20 - 30, sometimes after reaching 40 years of age. At the same time the structure of the trabecular meshwork becomes differentiated and hydrodynamic blocks can be associated with the anatomical features of the trabecular meshwork structure. Glaucoma in these cases has a relatively benign course that is observed in adult patients.

\section{Case History}

Patient D., 11 years old, has complex pathological changes of the anterior eye segment: megalocornea, corneal 
diameter of $13 \mathrm{~mm}$, posterior embryotoxon, front fixing of the iris, and the thickness of the iris stroma is 10 - 0 mkm (Figure 9a). Glaucoma developed at the age of 5.

D.'s brother was first examined 5 years ago at the age of 14. At that time the typical two-tone coloring of the iris was identified, the anterior chamber angle was open, the trabecula was partially covered with gray fibrous tissue, and the thickness of the iris stroma was 180 mkm (Figure 9b). Data for glaucoma for the period of examination and in the present time are not found.

\section{Conclusions}

Thus, the study of patients with inherited congenital mesenchymal dysgenesis and pathogenetic mechanisms of both the Frank-Kamenetsky Syndrome and glaucoma is allowed to determine the differences in the two clinical groups and to identify the criteria for early glaucoma development in patients with such syndrome.

Such criteria are a combination of:

1) Congenital subtotal atrophy of iris mesodermal layer (from 0 to $30 \mathrm{mkm}$ ) with signs of progressive dystrophy;

2) Nonprogressive congenital megalocornea (cornea diameter 12 - $14 \mathrm{~mm}$ );

3) Iridotrabecular dysgenesis of II-III degree;

4) Hyperopic refraction in axial myopia.

Thus, the formation of glaucoma in children is caused by dysgenesis of the anterior segment of the eye-a combination of congenital anomalies of the iris, cornea, and iridocorneal angle, which implies the inherited defects in the embryonic development of all germ layers of mesenchymal tissue.

\section{References}

[1] Becker, B. and Shaffer, R. (2004) Diagnosis and Therapy of the Glaucomas. Mosby, St. Louis.

[2] Frank-Kamenetskiy, Z.G. (1951) To the Question of Congenital Histoplasia of the Iris with Secondary Glaucoma. Proceedings of the Irkutsk Medical Institute, Dedicated to the 30th Anniversary of Its Existence, IGMI, Irkutsk, 281288.

[3] Frank-Kamenetskiy, Z.G (1925) The Peculiar Form of Hereditary Glaucoma. Russian Ophthalmological Journal, No. 3, 203-219.

[4] Brémond-Gignac, D. (2007) Glaucoma in Aniridia. Journal Français d'Ophtalmologie, 2, 196-199. http://dx.doi.org/10.1016/S0181-5512(07)89576-3

[5] Iureva, T.N. (2012) Mechanisms of Formation of Glaucoma Associated with Alteration of the Iris. Ph.D. Dissertation, Siberian Branch of the Russian Academy of Medical Sciences, Irkutsk.

[6] Levin, A.V. (2003) Congenital Eye Anomalies. Pediatric Clinics of North America, 1, 55-76. http://dx.doi.org/10.1016/S0031-3955(02)00113-X

[7] Apple, D.J. and Naumann, G.O.H. (1997) General Anatomy and Development of the Eye. Pathology of the Eye, 1-19.

[8] Radhakrishan, S., Rollins, A. and Roth, J. (2001) Real-Time Optical Coherence Tomography of the Anterior Segment at $1310 \mathrm{~nm}$. Archives of Ophthalmology, 119, 1179-1185. http://dx.doi.org/10.1001/archopht.119.8.1179

[9] Shchuko, A.G. and Iureva, T.N. (2009) Glaucoma and Pathology of the Iris. Borges, Moscow.

[10] Smelser, G.K. and Duke-Elder, S. (1990) Morphological and Functional Development of the Cornea. Symposium Abstract Book: The Transparency of the Cornea, Springfield, 23-39.

[11] Clemente, C.D. (2007) Anatomy: A Regional Atlas of the Human Body. Lippincott Williams \& Wilkins, Philadelphia.

[12] MacDonald, I.M., Tran, M. and Musarella, M.A. (2004) Ocular Genetics: Current Understanding. Survey of Ophthalmology, 2, 159-196. http://dx.doi.org/10.1016/j.survophthal.2003.12.003

[13] Deepak, P.E. and Kaufman, L.M. (2003) Anatomy, Development, and Physiology of the Visual System. Pediatric Clinics of North America, 1, 1-23.

[14] Idrees, F., Vaideanu, D. and Fraser, S.G. (2006) A Review of Anterior Segment Dysgeneses. Survey of Ophthalmology, 3, 213-231. http://dx.doi.org/10.1016/j.survophthal.2006.02.006

[15] Rodrigues, M.M., Jester, J.V. and Richards, R. (1985) Essential Iris Atrophy. A Clinical, Immunohistologic, and Electron Microscopic Study in an Enucleated Eye. Ophthalmology, 95, 69-73.

http://dx.doi.org/10.1016/S0161-6420(88)33234-3 